\title{
Suppression of electron spin decoherence in a quantum dot
}

\author{
Wenxian Zhang ${ }^{\dagger}$, V. V. Dobrovitski ${ }^{\dagger *}$, Lea F. Santos ${ }^{\ddagger}$, Lorenza Viola ${ }^{\ddagger}$, and B. N. Harmon ${ }^{\dagger}$ \\ $\dagger$ Ames Laboratory, Iowa State University, Ames, Iowa 50011, United States \\ $\ddagger$ Department of Physics and Astronomy, Dartmouth College, Hanover, New Hampshire 03755, United States \\ (Received 00 Month 200x; in final form 00 Month 200x)
}

\begin{abstract}
The dominant source of decoherence for an electron spin in a quantum dot is the hyperfine interaction with the surrounding bath of nuclear spins. The decoherence process may be slowed down by subjecting the electron spin to suitable sequences of external control pulses. We investigate the performance of a variety of dynamical decoupling protocols using exact numerical simulation. Emphasis is given to realistic pulse delays and the long-time limit, beyond the domain where available analytical approaches are guaranteed to work. Our results show that both deterministic and randomized protocols are capable to significantly prolong the electron coherence time, even when using control pulse separations substantially larger than what expected from the upper cutoff frequency of the coupling spectrum between the electron and the nuclear spins. In a realistic parameter range, the total width of such a coupling spectrum appears to be the physically relevant frequency scale affecting the overall quality of the decoupling.
\end{abstract}

\section{Introduction}

The dynamics of electron spins in semiconductor structures, such as quantum dots (QDs), has attracted much attention due to both its fundamental importance, as well as the potential applications in spinbased quantum information processing: not only does the electron spin degrees of freedom provide a natural two-level system with long energy relaxation time [1], but semiconductor-based quantum computing architectures can benefit from well-developed technology and a high degree of scalability [2]. Practical exploitation of such a potential, however, is severely hindered by the loss of phase coherence caused by the unavoidable coupling between the electron spin and its surrounding environment. For typical QD operating conditions and geometry, decoherence mechanisms associated with phonon processes or magnetic spin-orbit interactions are effectively suppressed, leaving the hyperfine coupling with nuclear spins as the dominant decoherence channel. The resulting decoherence dynamics occurs very rapidly, with the coherence times from ensemble measurements $\left(T_{2}^{*}\right)$ being of the order of tens of nanoseconds for typical GaAs QDs at experimentally relevant (sub-Kelvin) temperatures and moderate (sub-Tesla) magnetic fields [3].

As a result, viable methods for decoherence suppression are of key relevance. A number of proposals have been put forward for increasing the coherence time, for instance by seeking a high degree of nuclear spin polarization [5,4, by narrowing the nuclear spin distribution [6,7], or by cleverly disentangling the bath from the electron spin via external control [8]. However, these methods remain challenging to implement by current or near-term capabilities, and alternative approaches are still actively sought.

In this context, a promising approach is offered by dynamical decoupling (DD) methods [9]. Borrowing their inspiration from coherent averaging techniques in high-resolution NMR spectroscopy [10, 11, DD schemes aim at removing the effect of environmental coupling by subjecting the electron spin to suitably tailored sequences of control pulses. The long correlation time and distinctively non-Markovian behavior of the nuclear spin reservoir make the DD approach naturally suited for electron spin coherence control in QDs, as demonstrated by the recent implementation of single-pulse Hahn echo experiments [3, 12, which succeeded at extending $T_{2}^{*}$ by two orders of magnitude. Thus, it is both timely and important to undertake a comprehensive theoretical study of performance of multi-pulse DD protocols, in order to assess relative strengths and weaknesses, and identify optimal schemes. Only a few protocols have been

\footnotetext{
${ }^{*}$ Corresponding author. Email: slava@ameslab.gov

Journal of Modern Optics

ISSN 0950-0340 print/ISSN 1362-3044 online (c) 2007 Taylor \& Francis

http://www.tandf.co.uk/journals

DOI: $10.1080 / 0950034 \mathrm{YYXxxxxxxx}$
} 
quantitatively analyzed so far. In addition, the majority of investigations have focused on the case of QDs subjected to strong magnetic fields, predicting a coherence enhancement of at least an order of magnitude in GaAs QDs [13]. Still, the experimentally important situation of low-to-moderate fields is significantly more difficult to analyze due to the competition between purely adiabatic (energy-conserving) decoherence and relaxation processes. Existing studies have mostly relied on restrictive approximations - in particular, semi-classical treatments [14] or Magnus expansion (ME) techniques [15. Thus, little is known about the actual performance of DD protocols in a variety of practically important regimes.

Our goal in this paper is to further push a quantitative analysis of the electron spin decoherence problem in regimes which are relevant for experimental DD implementations in QDs, yet have received marginal consideration so far [16]. Special attention is devoted to the limit of zero external field, where dephasing and relaxation must be simultaneously eliminated, and the asymptotic long-time limit, where the effects of finite control rate and coherent error accumulation are crucial, so that neither the ME nor the standard quasi-static approximation for the nuclear spin bath are reliable a priori.

The content is organized as follows. Section 2 provides the essential background on the QD model Hamiltonian and various DD protocols. Section 3 summarizes both the relevant steps of the methodology used to tackle the problem, and presents a comparative analysis of all the DD protocols under investigation. A detailed discussion of the best DD protocol based on periodic concatenated design is presented, including the effects of control pulse delay, level of concatenation, intra-bath dipolar interaction, and coupling spectrum. Concluding remarks are given in Section 4.

\section{Quantum dot model and dynamical decoupling protocols}

The dynamics of an electron spin $S$ coupled to a bath $B$ of $N$ nuclear spins in a QD is described by a total Hamiltonian of the form

$$
\begin{gathered}
H=H_{S}+H_{S B}+H_{B} \\
H_{S}=\omega_{0} S_{z}, \quad H_{S B}=\mathbf{S} \cdot \sum_{k=1}^{N} A_{k} \mathbf{I}_{k}, \quad H_{B}=\sum_{k>l}^{N} \Gamma_{k l}\left(\mathbf{I}_{k} \cdot \mathbf{I}_{l}-3 I_{k}^{z} I_{l}^{z}\right) .
\end{gathered}
$$

Here, $\mathbf{S}$ and $\mathbf{I}_{k}$ denote the electron and the $k$-th bath spin operators, respectively; $\omega_{0}$ is the electron Zeeman energy in an external magnetic field $B_{0} ; H_{S B}$ gives the hyperfine contact interaction between the electron spin and the nuclei; and $H_{B}$ corresponds to the intra-bath dipolar coupling between nuclear spins [17, 18, 19, 12]. The parameters $A_{k}$ and $\Gamma_{k l}$ determine the strength of the respective interactions. In particular, the coupling energy per nucleus $A_{k}$ depends on the electron probability density at the nucleus location. We focus on the limit of zero external field $B_{0}=0$, so that all directions are equally affected by decoherence, and choose $I_{k}=1 / 2$, since the analysis does not depend qualitatively on the specific spin value (up to an appropriate renormalization of the parameters). The Zeeman energies of the nuclear spins and the anisotropy of the hyperfine coupling are very small and can be neglected for the time scales we are interested in. The bath is assumed to be initially unpolarized, which is justified by the fact that in typical experiments the temperature is much larger than the characteristic energy of nuclear spins.

In the absence of control pulses, the electron spin described by the above Hamiltonian undergoes free induction decay (FID) on a time scale given by $T_{2}^{*}=\left(N A^{2} / 8\right)^{-1 / 2}$, where $A=\left(\sum_{k} A_{k}^{2} / N\right)^{1 / 2}$ is approximately $10^{-4} \mu \mathrm{eV}$ for typical GaAs QDs with $N=10^{6}[19$. In our analysis, we set $\hbar=1$, and measure frequency and energy in units of $A$, with time correspondingly being given in units of $1 / A$.

In order to slow down the decoherence process, we recur to DD methods. The basic idea consists of modifying the system dynamics by subjecting the electron spin to a sequence of $\pi_{\hat{\mathbf{n}}}$ pulses, where $\hat{\mathbf{n}}$ denotes the control axis. In the simplest setting, the pulses are equally separated by an interval $\tau$, during which the system evolves freely. We assume bang-bang controls, that is, arbitrarily strong and instantaneous pulses, which we draw from a control group $\mathcal{G}=\left\{g_{j}\right\}, j=0, \ldots,|\mathcal{G}|-1$, according to $P_{i, j}=g_{j} g_{i}^{\dagger}$. For a single spin as relevant here, the sequences are derived from the irreducible Pauli group $\mathcal{G}_{P}=\{I, X, Y, Z\}$ ] , where $I, X, Y, Z$ are the identity and the Pauli matrices of the electron spin, respectively. A variety of control protocols have been developed, which exploit deterministic [9, 15] and stochastic [20,21] design. In cyclic 
DD methods, the pulse sequence is fixed, pre-determined, and periodically repeated in time; in randomized DD, the future control actions are not known in advance, but chosen at random from a set of alternatives.

The construction of deterministic DD sequences has been mainly based on the average Hamiltonian theory [11], having as a goal the cancellation of the dominant terms in the ME series for the underlying time evolution operator. The simplest periodic DD (PDD) sequence we consider here ensures the removal of the zeroth order term $\overline{\mathcal{H}}^{(0)}$ of the average Hamiltonian $\overline{\mathcal{H}}$ at every $T_{n}=n T_{c}=n|\mathcal{G}| \tau$, where $n \in \mathbb{N}, T_{c}$ is the cycle time, and $|\mathcal{G}|=4$ is the size of the DD group. By fixing the path of $\mathcal{G}_{P}$ as $\{I, X, Y, Z\}$, the control sequence becomes $C_{1}=C_{0} X C_{0} Z C_{0} X C_{0} Z$, where $C_{0}$ denotes the interval of free evolution. Thus, the cycle propagator for a system subjected to the PDD sequence is given by

$$
\begin{aligned}
U\left(T_{c}\right)=\mathcal{T} \exp \left\{-i \int_{0}^{T_{c}}\left[H+H_{c}(s)\right] d s\right\} & \equiv \exp \left[-i \overline{\mathcal{H}} T_{c}\right]=\exp \left[-i\left(\overline{\mathcal{H}}^{(0)}+\overline{\mathcal{H}}^{(1)}+\cdots\right) T_{c}\right] \\
& =Z U_{0} X U_{0} Z U_{0} X U_{0},
\end{aligned}
$$

where $\mathcal{T}$ indicates time ordering, $H_{c}(t)$ corresponds to the applied control Hamiltonian, $U_{0}=\exp (-i H \tau)$ is the free propagator, and the zeroth order term in the ME is

$$
\overline{\mathcal{H}}^{(0)}=\frac{1}{|\mathcal{G}|} \sum_{k} g_{k}^{\dagger} U_{0}\left(t_{k+1}, t_{k}\right) g_{k}=\frac{1}{4}[Z H Z+Y H Y+X H X+H]=H_{B},
$$

that is, terms coupling the system and bath degrees of freedom are removed to lowest order. The terms depending on $H_{S B}$ appear in the higher-order corrections, in particular $\overline{\mathcal{H}} \sim \overline{\mathcal{H}}^{(1)} \propto \tau$, which guarantees DD between the system and the bath in the limit of arbitrarily fast control, $\tau \rightarrow 0$. Clearly, $\tau$ is necessarily finite in realistic settings, its minimum value being constrained by practical limitations (e.g., for QDs and other devices operating at dilution refrigerator temperatures, the need to avoid excessive radio-frequency heating of the sample, which imposes limitation on the total absorbed power). In order to improve DD performance, going beyond what is achievable by the first-order schemes above, two main strategies are available: higher-order DD, ensuring cancellation of higher order terms in the effective Hamiltonian; and reduction of error accumulation in time due to the remaining terms of the Hamiltonian.

As prototypes of higher-level deterministic sequences, we consider symmetric DD (SDD) and concatenated DD (CDD) [15]. The SDD sequence corresponds to a symmetrization of $C_{1}$, being written as $C_{S}=C_{0} X C_{0} Z C_{0} X C_{0} I C_{0} X C_{0} Z C_{0} X C_{0}$. Its cycle time is twice as long as PDD, but at every $T_{n}=2 n T_{c}=8 n \tau$, besides $\overline{\mathcal{H}}^{(0)}$, all odd terms in $\overline{\mathcal{H}}$ are also canceled, leading to $\overline{\mathcal{H}} \propto \tau^{2}$. CDD relies on a temporal recursive structure, so that at level $\ell+1$ the pulse sequence is given by $C_{\ell+1}=C_{\ell} X C_{\ell} Z C_{\ell} X C_{\ell} Z$. Here, we truncate the concatenation procedure at a certain level and repeat a periodic sequence, referred to as $\mathrm{PCDD}_{\ell}$, after every $4^{\ell} \tau$. For example, $\ell=2$ leads to $\mathrm{PCDD}_{2}$, which is also a symmetric protocol, although usually superior than SDD at reducing error accumulation.

The main goal of stochastic protocols is to guarantee that errors accumulate probabilistically in time, instead of coherently as in deterministic sequences. As representatives of these methods, we consider naive random DD (NRD), random path DD (RPD), and symmetrized random path DD (SRPD). NRD corresponds to a sequence of pulses chosen randomly from $\mathcal{G}_{P}$ with equal probability. For RPD, randomization is associated with the path to traverse the group $\mathcal{G}_{P}$, that is, at every $T_{n}=4 \tau$ one chooses at random one of the following options: $\left\{C_{0} X C_{0} Y C_{0} X C_{0} Y, C_{0} X C_{0} Z C_{0} X C_{0} Z, C_{0} Y C_{0} X C_{0} Y C_{0} X, C_{0} Y C_{0} Z C_{0} Y C_{0} Z\right.$, $\left.C_{0} Z C_{0} X C_{0} Z C_{0} X, C_{0} Z C_{0} Y C_{0} Z C_{0} Y\right\}$. SRPD improves over RPD by symmetrizing the random paths as in SDD and inserting a random pulse between paths. Besides slowing down the error accumulation at long times, RPD and SRPD also ensure good performance at short times, since they lead to effective Hamiltonians $H_{\text {eff }} \propto \tau$ and $H_{\text {eff }} \propto \tau^{2}$, respectively.

Note that for randomized DD the periodicity, as well as the notion of a control cycle, is lost in general. Thus, the comparison between deterministic and randomized protocols is most naturally carried out in the so-called logical frame. In this frame, $H_{c}(t)$ is removed from the evolution through the transformation $\tilde{U}(T)=U_{c}^{\dagger}(T) U(T)$, where tilde indicates logical frame, $U_{c}(T)=\mathcal{T} \exp \left[-i \int_{0}^{T} H_{c}(s) d s\right]$ corresponds to the 
control propagator, and $U(T)$ is the propagator in the physical frame, as given by Eq. (3). For deterministic sequences, both physical and logical propagators stroboscopically coincide at the completion of each cycle, but the same is not necessarily true for the stochastic methods. In the latter case, in order to return the state of the system to the physical frame, it suffices to keep track of the applied control pulses.

\section{Performance of control protocols}

\subsection{Control metric}

To compare the above-mentioned control protocols in a way which avoids dependence on the initial state of the systems, $\left|\psi_{S}(0)\right\rangle$, we shall consider the worst-case scenario and compute the minimum input-output pure-state fidelity,

$$
F_{m}(T)=\min _{\left|\psi_{S}(0)\right\rangle} \operatorname{Tr}\left[\tilde{\rho}_{S}(T) \rho_{S}(0)\right]
$$

where $\tilde{\rho}_{S}(T)$ corresponds to the reduced density matrix of the electron spin at time $T$. Equivalently, $F_{m}(T)$ may be thought of as the minimum state overlap between the intended and the decoupled system evolution.

Analytical bounds on the worst-case pure-state expected fidelity have been established for various DD protocols [20,21, 15, however they apply only to short evolution times and small pulse separations. Numerical simulations are mandatory to investigate DD performance at long times and for a broad range of $\tau$ values. We are especially interested in pulse delays beyond the restricted domain where the ME is guaranteed to converge, $\omega_{c} T_{c} \ll 1$, where $\omega_{c}$ denotes a high frequency cutoff. While in most situations $\omega_{c}$ is determined by $H_{B}$, for the QD problem the nuclear spin bath is to a very high accuracy non-dynamical, thus $\omega_{c}$ is determined by the electron-nuclear coupling Hamiltonian $H_{S B}$. This leads to $\omega_{c} \approx \sum_{k}\left|A_{k}\right| / 4 \sim N A / 4$, which is very large for typical QDs. We will consider $\tau \sim 1 / 2 \sigma$, where $\sigma$ represents the power spectrum width of the entire system, with $2 \sigma \approx\left(\sum_{k} A_{k}^{2}\right)^{1 / 2}=\sqrt{N} A$ [22]. Thus, the basic cycle time $T_{c}=4 \tau \sim \sqrt{N} \omega_{c}^{-1}$ will be up to a factor $\sqrt{N}$ longer than the formal (worst-case) convergence requirement of the ME. We shall also assume that $A_{k}>0$ are uniformly distributed random numbers.

The minimum fidelity is numerically obtained by employing the quantum process tomography method 23] as follows. The initial state of the entire system is taken as a direct product of the electron spin state $\left|\psi_{S}(0)\right\rangle$ and the bath spin state $\left|\psi_{B}(0)\right\rangle$. Four different $\left|\psi_{S}(0)\right\rangle$ are considered: $|\uparrow\rangle,|\downarrow\rangle,(|\uparrow\rangle+|\downarrow\rangle) / \sqrt{2}$, and $(|\uparrow\rangle+i|\downarrow\rangle) / \sqrt{2}$; while a single $\left|\psi_{B}(0)\right\rangle$ is assumed, corresponding to a random superposition $\left|\psi_{B}(0)\right\rangle=\sum_{i=1}^{2^{N}} c_{i}\left|\phi_{i}\right\rangle$ of all possible tensor products of the form $|\phi\rangle=|\uparrow\rangle_{1} \otimes|\downarrow\rangle_{2} \otimes|\downarrow\rangle_{3} \otimes \ldots \otimes|\uparrow\rangle_{N}$, where $c_{i}$ are uniform random numbers. The time-dependent Schrödinger equation for the joint system plus bath dynamics is then solved by applying the Chebyshev polynomial expansion method to the evolution operator [18. At final time $T$, a partial trace over the bath is performed, and the four resulting reduced density matrices are used to compute the $4 \times 4 \chi$-matrix 23 . The $\chi$-matrix is a superoperator that describes the effective dynamics of the electron spin and allows for the reduced density matrix of an arbitrary electron initial state to be evaluated from the equation

$$
\tilde{\rho}_{S}(T)=\sum_{m, n=0}^{3} K_{m} \rho_{S}(0) K_{n}^{\dagger} \chi_{m n}(T)
$$

where $K_{0}=I, K_{1}=X, K_{2}=-i Y$, and $K_{3}=Z$. Since an arbitrary initial state of $S$ in the Bloch sphere may be expressed as $\left|\psi_{S}(0)\right\rangle=\cos (\theta / 2)|\uparrow\rangle+\sin (\theta / 2) e^{i \varphi}|\downarrow\rangle, \theta \in[0, \pi], \varphi \in[0,2 \pi], F_{m}(T)$ simplifies to

$$
F_{m}(T)=\min _{(\theta, \varphi)}\left\langle\psi_{S}(0)\left|\tilde{\rho}_{S}(T)\right| \psi_{S}(0)\right\rangle .
$$

In practice, after determining the matrix $\chi$, we obtain $F_{m}(T)$ from numerical minimization, by comparing the results over a statistically meaningful set of initial guesses for $(\theta, \varphi)$, and then selecting the worst case. 


\subsection{Results}

Fig. 1(a) shows $F_{m}(T)$ for the above-mentioned DD protocols. For comparison, we plot also the FID signal, for which no control is applied. Because the characteristic time scale $\tau_{D}$ for the nuclear dipolar dynamics (resulting from $H_{B}$ ) is at least two orders of magnitude slower than the one due to $H_{S B}$ in typical QDs, setting $H_{B}=0$ is justified over the time scales studied here. As seen, all schemes lead to substantial enhancement of the electron spin coherence, provided that the control time scale is sufficiently fast compared to an effective upper cutoff determined by the spectral width $2 \sigma$. Similar to the behavior observed for DD of $1 / f$ noise [24], this indicates how general convergence arguments may place too stringent a bound for realistic systems, actual performance depending sensitively on spectral details.

Among the various protocols, NRD shows (not surprisingly) the worst performance, consistent with the expectation that its advantages over deterministic DD appear only when the control group is very large. PDD and SDD are, respectively, outperformed by RPD and SRPD, confirming the reduction of error accumulation associated with randomization. Interestingly, the RPD protocol provides worse fidelity than SDD at times smaller than 10, but eventually also outperforms SDD at longer times. The best performance for this system, however, is achieved by the deterministic $\mathrm{PCDD}_{2}$. Compared with the case of closed systems under the action of reducible control groups investigated in [21, this confirms how concatenated design is especially efficient for irreducible averaging. A feature which also proves beneficial for concatenation is the absence of internal bath dynamics [15].
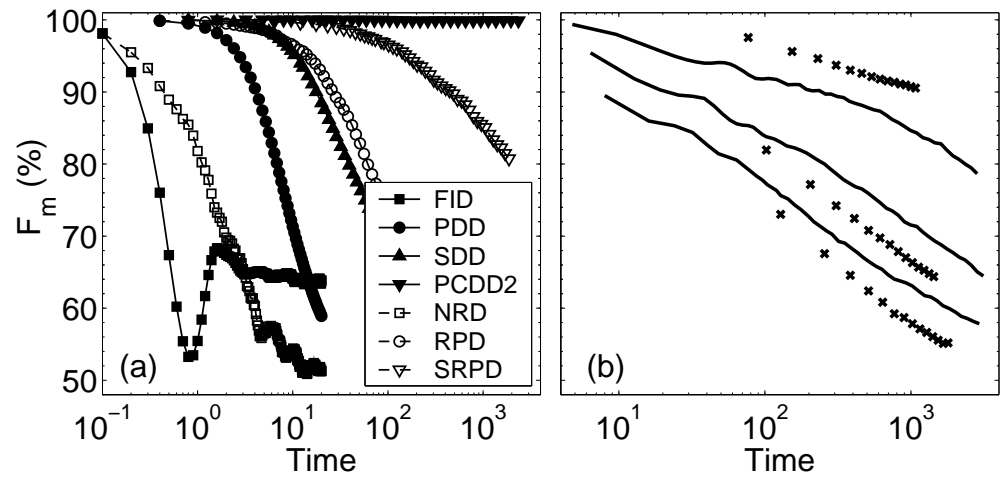

Figure 1. Minimum fidelity vs. time in the logical frame. The Hamiltonian parameters are $B_{0}=0, \Gamma_{0}=0$, and $N=15$. (a) Comparison of various protocols with $\tau=0.1$. For NRD and FID, data points are acquired after every $\tau$, for deterministic DD (PDD, $\mathrm{SDD}, \mathrm{PCDD}_{2}$ ), right at the completion of each cycle, while for RPD and SRPD this is done after every $4 \tau$ and $8 \tau$, respectively. Random protocols are averaged over $10^{2}$ control realizations. (b) Comparison of $\mathrm{PCDD}_{2}$ (solid lines) and PCDD 4 (crosses) for $\tau=0.3$, $0.4,0.5$ from top to bottom.

The above findings prompt a more in-depth analysis of the PCDD protocol. In Fig. 1(b), we compare the performance of two levels of concatenation, $\ell=2,4$, for different values of $\tau$. As expected, the quality deteriorates as $\tau$ increases but, unexpectedly, $\mathrm{PCDD}_{4}$ underperforms $\mathrm{PCDD}_{2}$ for larger $\tau$, suggesting that predictions based on applications of the ME [15] may not hold in this case.

The dependence of the $\mathrm{PCDD}_{2}$ performance on the pulse delay $\tau$ is investigated in Fig. 2(a), by focusing on the time $T_{90 \%}$ where the protocol attains $F_{m}\left(T_{90 \%}\right)=0.9$ for given $\tau$. Two main features are observed: (i) $T_{90 \%}$ increases monotonically as $\tau$ decreases - consistent with the fact that no crossover from decoherence suppression to decoherence acceleration is expected as long as $\tau$ is sufficiently small with respect to the inverse spectral width of the environmental coupling [9, 24]. That is, in terms of the formal analogy established between decoherence control methods based on DD pulses and frequent quantum measurements [25, 26], only the counterpart of quantum Zeno effect (QZE) is observed in the relevant parameter regime, with no occurrence of "anti-Zeno" behavior; (ii) The $\mathrm{PCCD}_{2}$ performance depends quite sensitively on $\tau$, moderate changes in the value of $\tau$ (say, by a factor of two) changing the coherence time $T_{90 \%}$ by up to three orders of magnitude. For $\mathrm{PCDD}_{2}$, the leading non-zero term in the $\mathrm{ME}$ is $\overline{\mathcal{H}}^{(2)}$, so that the error amplitude per cycle is bounded by $\mathcal{O}\left(|\mathcal{G}|^{3}(\sigma \tau)^{3}\right)$, and therefore the total error amplitude after a number $T_{90 \%} /(|\mathcal{G}| \tau)$ of cycles grows as $\mathcal{O}\left(T_{90 \%} \sigma^{3}(|\mathcal{G}| \tau)^{2}\right)$. As a result, within the regime of validity of the ME, the time $T_{90 \%}$ at which the total error probability $1-F_{m}\left(T_{90 \%}\right)$ reaches $10 \%$ is expected to increase 

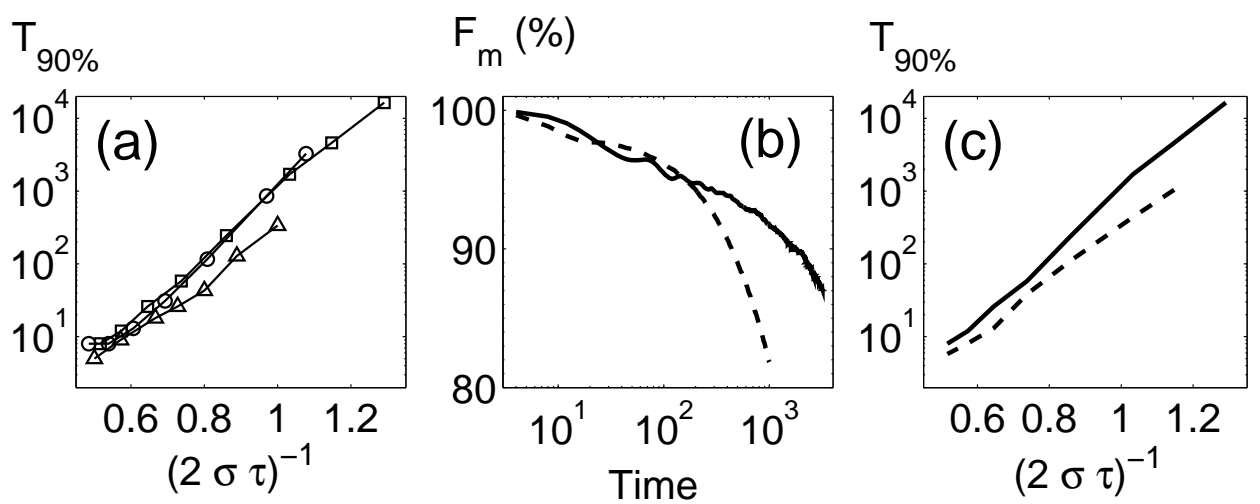

Figure 2. (a) $T_{90 \%}$ vs. $\tau$ : Comparison of $\mathrm{PCDD}_{2}$ for bath sizes $N=15$ (squares), 17 (circles), and 25 (triangles); $H_{B}=0$. The time $T_{90 \%}$ depends strongly on $\tau$, varying by three orders of magnitude when $\tau$ is varied by a factor of two. (b) The effect of dipolar interaction between nuclear spins on $\mathrm{PCDD}_{2}$ performance, $F_{m}$ vs. time for $\tau=0.25$; (c) $T_{90 \%} v s$. $\tau$. For both (b) and (c), the parameters are $N=15, B_{0}=0$, and uniformly distributed random numbers $\Gamma_{k l} \in\left[-\Gamma_{0}, \Gamma_{0}\right] ; \Gamma_{0}=0$ (solid line), $\Gamma_{0}=0.1$ (dashed line).

as $1 / \tau^{2}$ with decreasing $\tau$. The steeper dependence shown by the data in Fig. 2(a) indicates that a naive extrapolation of the above estimate in the long-time regime of relevance is not directly viable.

Finally, we investigate the effect of interaction between the bath spins, taking the intra-bath coupling parameters $\Gamma_{k l}$ in (1) as uniformly distributed random numbers in $\left[-\Gamma_{0}, \Gamma_{0}\right]$, and assuming that the bath spins are located on a $3 \times 5$ piece of a two-dimension square lattice. One may expect that the effect of intra-bath coupling becomes important once the characteristic coupling strength $\Gamma_{0}$ is comparable with the system-bath coupling $A$. However, Figs. 2(b) and (c) show that the effect of intra-bath interactions becomes clearly visible already for much smaller values of $\Gamma_{0}=0.1 A$, leading to a decrease of the coherence time $T_{90 \%}$ by an order of magnitude in comparison with the case $\Gamma_{0}=0$. Although the fast bath dynamics regime is not directly relevant to standard GaAs QDs, where $\Gamma_{0} \sim 0.01 A$, our observations may be useful as a first step towards characterization of DD in other systems.

\subsection{Coupling spectrum}

Additional physical insight into the performance of DD schemes may be gained by considering the coupling spectrum of the QD Hamiltonian (10) in the absence and in the presence of control. A typical spectrum for uniformly distributed random $A_{k}$ (obtained numerically) is shown in Fig. 3(a). For the homogeneous case of constant $A_{k}=A$ (below, we set $A=1$ for convenience), the spectrum may be evaluated analytically, the result being also shown in Fig. 3(a). Physically, the limit $A_{k}=A$ is equivalent to a semiclassical treatment of the bath, where the quantum-mechanical operator $\mathbf{B}=\sum_{k} A_{k} \mathbf{I}_{\mathbf{k}}$, representing the Overhauser field exerted by the bath on the electron spin, is approximated by a randomly oriented classical vector $\mathbf{B}$ with zero mean and variance $\sigma$ [14, 19, 12].

For $A_{k}=A$, the operator of the total bath spin $\mathbf{I}=\sum_{k} \mathbf{I}_{k}$ is an integral of motion, and the Hamiltonian (11) acquires a simple form of two coupled spins,

$$
H=A \mathbf{S} \cdot \mathbf{I}=H_{\text {diag }}+H_{o f f}=A S_{z} I_{z}+A\left(S_{x} I_{x}+S_{y} I_{y}\right),
$$

where $I \gg 1$. Let us consider a state of the whole system $\left|\uparrow ; I, I_{z}\right\rangle$, which corresponds to the electron spin up, and the bath having total spin $I$ with $z$-projection equal to $I_{z}$. Since the value of $I_{z}+S_{z}$ is also an integral of motion, this state is coupled only to one state, $\left|\downarrow ; I, I_{z}+1\right\rangle$. Thus, the total Hamiltonian matrix can be separated into a number of uncoupled $2 \times 2$ blocks, each block involving a pair of levels $\left|\uparrow ; I, I_{z}\right\rangle$ and $\left|\downarrow ; I, I_{z}+1\right\rangle$. Correspondingly, the whole system can be viewed as a collection of uncoupled two-level systems, with state space spanned by basis states $\left|\uparrow ; I, I_{z}\right\rangle$ and $\left|\downarrow ; I, I_{z}+1\right\rangle$. The energy splitting between these basis states, $\epsilon=I_{z}+1 / 2$, is determined by the diagonal part of the Hamiltonian (8), $H_{\text {diag }}$, whereas the coupling matrix element between the basis states is determined by the non-diagonal part $H_{\text {off } f}$.

The coupling spectrum $G(\omega)$ quantifies the density of bath states weighted by the corresponding coupling 

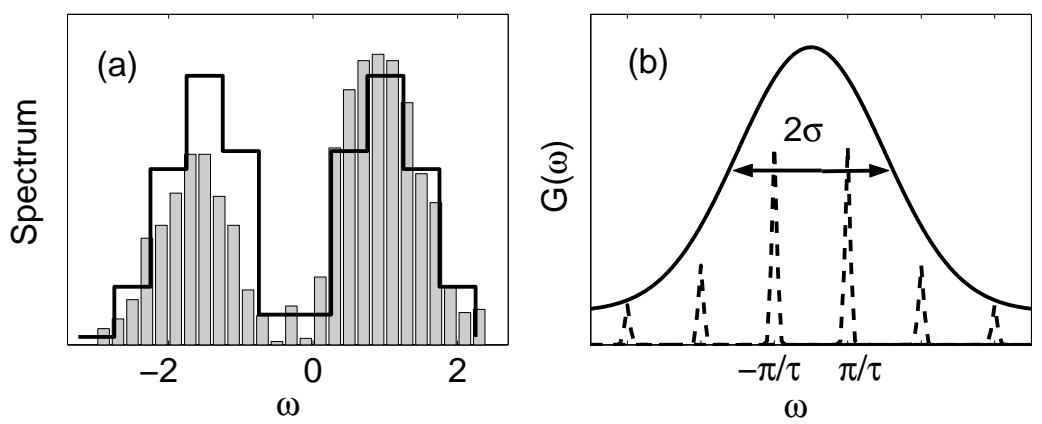

Figure 3. (a) Spectrum of the QD for $B_{0}=0, \Gamma_{0}=0$, and $N=10$. The bars are obtained for a single realization of random $A_{k}$, whereas the solid line corresponds to the homogeneous limit, $A_{k}=A$. (b) Pictorial representation of the coupling spectrum $G(\omega)$ (solid line) and the control pulse spectrum (dashed lines).

strength. The spectral density $n(\omega)$ of two-level systems having energy splitting $\epsilon=\omega$ may be computed by a combinatorial argument: this is the density of the bath states having a given value of $I_{z}$ (since $\epsilon=I_{z}$ for $I_{z} \gg 1$ ), which leads to

$$
n(\omega)=\frac{1}{\sqrt{2 \pi} \sigma} \exp \left(-\omega^{2} / 2 \sigma^{2}\right)
$$

The modulus square of the matrix element $V$ which couples the basis states $\left|\uparrow ; I, I_{z}\right\rangle$ and $\left|\downarrow ; I, I_{z}+1\right\rangle$ is $|V|^{2}=\left(A^{2} / 4\right)\left(I^{2}-I_{z}^{2}\right)$, and it should be averaged over all two-level systems having the same $I_{z}$ (i.e., the same $\omega$ ) but different values of $I$. We then find that $|V|^{2}=\sigma^{2} / 2$, and the coupling spectrum $G(\omega)$ for the electron spin plus bath is (to a good accuracy) a sum over all coupling spectra of the individual two-level systems [27],

$$
G(\omega)=|V|^{2} n(\omega)=\frac{\sigma}{2 \sqrt{2 \pi}} \exp \left(-\omega^{2} / 2 \sigma^{2}\right)
$$

Control modifies, in general, the above coupling spectrum. Under DD, in particular, the relevant spectrum becomes a convolution of the original coupling spectrum $G(\omega)$ with the spectrum of the control pulses (Fig. 3(b)), which is given by a series of $\delta$-like peaks separated by a distance of the order of $1 / \tau$ [25]. The two central peaks of the controlled spectrum have the weight of $4 / \pi^{2}$ [25], while the contribution from the remaining peaks is less than $20 \%$ and thus may be omitted. As a result, in the presence of DD pulses, the decay is caused predominantly by the part of $G(\omega)$ which has $\omega \sim \pi / \tau$. Such a contribution decreases exponentially, as $\exp \left(-\omega^{2} / 2 \sigma^{2}\right)$, with decreasing $\tau$. Correspondingly, DD becomes efficient for $\tau \sim 1 / \sigma$, the inverse width of the spectrum, not the inverse spectrum cutoff frequency $\omega_{c}$ (which is larger than $\sigma$ by a factor of $\sqrt{N} \gg 1)$.

The fast decrease of $G(\omega)$ with $\omega$ may provide an intuition as to why $T_{90 \%}$ depends so drastically on $\tau$, as seen in Fig. 2(a). If we assume, in analogy with [24,25], that the time scale $T_{90 \%}$ is inversely proportional to the contribution of $G(\omega)$ at $\omega=q \pi / \tau$ (where $q$ is some protocol-dependent parameter of the order of unity), then a strong dependence on $\tau$ is indeed expected:

$$
T_{90 \%} \sim \frac{2 \sqrt{2 \pi}}{\sigma} \exp \left(q^{2} \pi^{2} / 2 \sigma^{2} \tau^{2}\right)
$$

Also, Eq. (10) further demonstrates why the anti-Zeno-like behavior (acceleration of decoherence by DD pulses) is not observed in our simulations: $G(\omega)$ monotonically decreases with $\omega$, and even for large $T_{c}$ the total power of the modified coupling spectrum cannot increase. Since, as noted, our results for $N=15$, 17, and 25 (Fig. 2(a)) are very close to each other, we expect that the basic qualitative features of our analysis remain applicable to realistic situations with large $N$. 


\section{Conclusion}

We have characterized quantitatively the performance of various DD protocols for an electron spin coupled to a nuclear spin bath, including both standard cyclic and randomized DD design. Two main points emerge from our analysis: on one hand, DD methods demonstrate a clear potential for significantly enhancing the electron spin coherence time for an arbitrary initial state, actual performance depending on both control and physical parameters. In particular, the frequency spectrum of the environmental coupling proves fundamental for identifying the minimum time scale requirements that must be met in order for DD to be effective. On the other hand, care must be taken in extrapolating simplified approaches (such as the ME) to parameter regimes outside the established domain of applicability, in particular for coherence preservation over long times. While additional investigation is needed to gain a complete picture (including, for instance, the effect of realistic control imperfections), it is our hope that the steady progress witnessed in experimental solid-state control techniques [28,29] may soon allow to validate the usefulness of DD in realistic QD devices.

\section{Acknowledgement}

It is a pleasure to thank D. G. Cory, A. Imamoglu, J. J. Longdell, and A. J. Rimberg for discussions. This work was supported by the Department of Energy, Basic Energy Sciences, under Contract No. DEAC02-07CH11358. Work at Ames was also supported by the NSA and ARDA under ARO contract DAAD 19-03-1-0132. L. V. also acknowledges partial support from the NSF through Grant No. PHY-0555417.

\section{References}

[1] J. M. Elzerman et al. , Nature (London) 430431 (2004).

[2] D. Loss and D. P. DiVincenzo, Phys. Rev. A 57120 (1998).

[3] A. C. Johnson et al., Nature (London) 435925 (2005); F. H. L. Koppens et al., Science 3091346 (2005); J. R. Petta et al., ibid. 3092180 (2005).

[4] G. Burkard, D. Loss, and D. P. DiVincenzo, Phys. Rev. B 592070 (1999).

[5] A. Imamoğlu, E. Knill, L. Tian, and P. Zoller, Phys. Rev. Lett. 91017402 (2003).

[6] W. A. Coish and D. Loss, Phys. Rev. B 70, 195340 (2004); D. Stepanenko, G. Burkard, G. Giedke, and A. Imamoglu, Phys. Rev. Lett. 96136401 (2006); D. Klauser, W. A. Coish, and D. Loss, Phys. Rev. B 73, 205302 (2006).

[7] G. Giedke, J. M. Taylor, D. D’Alessandro, M. Lukin, and A. Imamoğlu, Phys. Rev. A 74032316 (2006).

[8] W. Yao, R.-B. Liu, and L. J. Sham, Phys. Rev. Lett. 98077602 (2007).

[9] L. Viola and S. Lloyd, Phys. Rev. A 582733 (1998); L. Viola, E. Knill, and S. Lloyd, Phys. Rev. Lett. 822417 (1999).

[10] M. Mehring, Principles of High Resolution NMR in Solids (Springer-Verlag, Berlin, 1983).

[11] B. C. Gerstein and C. R. Dybowski, Transient Techniques in NMR of Solids: An Introduction to Theory and Practice (Academic Press, Orlando, Florida, 1985).

[12] J. M. Taylor et al., eprint cond-mat/0602470

[13] W. M. Witzel, R. de Sousa, and S. Das Sarma, Phys. Rev. B $72161306($ R) (2005); W. M. Witzel and S. Das Sarma, ibid. 74 035322 (2006); W. Yao, R.-B. Liu, and L. J. Sham, ibid. 74195301 (2006); N. Shenvi, R. de Sousa, and K. B. Whaley, ibid. 71224411 (2005); W. M. Witzel and S. Das Sarma, Phys. Rev. Lett. 98077601 (2007).

[14] J. Bergli and L. Glazman, eprint cond-mat/0609490

[15] K. Khodjasteh and D. A. Lidar, Phys. Rev. Lett. 95180501 (2005); eprint quant-ph/0607086

[16] W. Zhang, V. V. Dobrovitski, L. F. Santos, L. Viola, and B. N. Harmon, eprint cond-mat/0701507

[17] K. A. Al-Hassanieh, V. V. Dobrovitski, E. Dagotto, and B. N. Harmon, Phys. Rev. Lett. 97037204 (2006).

[18] V. V. Dobrovitski and H. A. De Raedt, Phys. Rev. E 67056702 (2003); W. Zhang, N. Konstantinidis, K. A. Al-Hassanieh, and V. V. Dobrovitski, J. Phys.: Condens. Matter 19083202 (2007).

[19] W. Zhang, V. V. Dobrovitski, K. A. Al-Hassanieh, E. Dagotto, and B. N. Harmon, Phys. Rev. B 74205313 (2006).

[20] L. Viola and E. Knill, Phys. Rev. Lett. 94060502 (2005); O. Kern and G. Alber, ibid. 95250501 (2005).

[21] L. F. Santos and L. Viola, Phys. Rev. Lett. 97150501 (2006).

[22] A. Melikidze, V. V. Dobrovitski, H. A. De Raedt, M. I. Katsnelson and B. N. Harmon, Phys. Rev. B 70014435 (2004).

[23] M. A. Nielsen and I. L. Chuang, Quantum Computation and Quantum Information (Cambridge University Press, Cambridge, 2002).

[24] K. Shiokawa and D. A. Lidar, Phys. Rev. A 69 030302(R) (2004); L. Faoro and L. Viola, Phys. Rev. Lett. 92 117905 (2004); G. Falci, A. D'Arrigo, A. Mastellone, and E. Paladino, Phys. Rev. A 70 040101(R) (2004).

[25] A. G. Kofman and G. Kurizki, Nature (London) 405546 (2000); ibid., Phys. Rev. Lett. 87270405 (2001).

[26] P. Facchi, D. A. Lidar, S. Pascazio, Phys. Rev. A 69032314 (2004); P. Facchi et al., Phys. Rev. A 71022302 (2005).

[27] This expression may not apply for extremely small $\omega$, but the contribution from such part of the spectrum is negligible.

[28] F. H. L. Koppens et al., Nature (London) 442766 (2006); P. Chen et al., Phys. Rev. B 69075320 (2004); A. Greilich et al., Phys. Rev. Lett. 96227401 (2006); M. V. G. Dutt et al., Phys. Rev. B 74125306 (2006); C. Emary and L. J. Sham, eprint cond-mat/0608518

[29] E. Fraval, M. J. Sellars, and J. J. Longdell, Phys. Rev. Lett. 92077601 (2004); ibid 95030506 (2005). 